\title{
Técnica de Marsupialização em Rânula: Relato de caso
}

\author{
Marsupialization Technique in Ranula: Case Report
}

Tecnica de Marsupialización en Ranula: Relato de caso

\begin{abstract}
Fabiana Peixoto Gomes ${ }^{1 *}$, Isabelle Costa de Almeida Percianoㄹ, Diego Maurício de Oliveira ${ }^{1}$, Bruna Tatiane Martins dos Santos ${ }^{1}$, Laryssa Costa Canuto ${ }^{1}$, Ana Luiza Pontes de Oliveira', Fernanda Braga Peixoto'1.
\end{abstract}

\section{RESUMO}

Objetivo: Relatar o caso clínico de uma paciente diagnosticada com rânula e a técnica de tratamento proposto, onde o mesmo mostrou resolutividade para o caso. Detalhamento do caso: Paciente, sexo feminino, 23 anos, compareceu ao serviço, com queixa principal "tenho uma bolha na boca que desaparece e volta, e ela não dói". $\mathrm{Na}$ história da doença atual, a paciente se queixa de lesão em formato de bolha, que surgiu há aproximadamente seis meses, na qual surge e desaparece frequentemente, sem causa aparente e sendo esta lesão indolor. Ao exame intra oral foi observado uma lesão bolhosa, única, de cor violácea, superfície lisa, limites nítidos, formato ovalado, flutuante, indolor e localizada no assoalho bucal. A hipótese de diagnóstico foi de Rânula. Realizou-se marsupialização da lesão e confirmação da lesão posterior resultado do histopatológico. Considerações finais: Por ser diagnóstico diferencial de outras lesões, foi realizado histopatológico para um correto diagnóstico e execução do tratamento mais adequado para o caso, que seria a cirurgia para remoção do teto da lesão, sendo esse procedimento o que possui melhor prognóstico. É de grande importância que o Cirurgião-dentista obtenha conhecimento para uma melhor conduta do tratamento e sobretudo, sobrevida do paciente.

Palavras-chave: Mucocele, Rânula, Patologia bucal.

\begin{abstract}
Objective: Report the clinical case of a patient diagnosed with rancula and the proposed treatment technique, where it showed resolution for the case. Case detailing: Female patient, 23 years old, attended the service, with the main complaint "I have a bubble in my mouth that disappears and comes back, and it doesn't hurt". In the history of the current illness, the patient complains of a blister-shaped lesion, which appeared approximately six months ago, in which it often appears and disappears without apparent cause and is painless. On intra-oral examination, a single bullous lesion of violet color, smooth surface, clear boundaries, oval shape, floating, painless and located on the buccal floor were observed. The hypothesis of diagnosis was of Ranula. The lesion marsupialization was performed and the subsequent lesion confirmed by histopathology. Final considerations: Because it is a differential diagnosis of other lesions, histopathology was performed for a correct diagnosis and execution of the most appropriate treatment for the case, which would be surgery to remove the roof of the lesion, which procedure has the best prognosis. It is of great importance that the dental surgeon gain knowledge for a better treatment conduct and especially patient survival.
\end{abstract}

Keywords: Mucocele, Ranula, Oral pathology.

${ }^{1}$ Centro Universitário Cesmac, Maceió-Alagoas. *E-mail: fabbipeixoto@hotmail.com

SUBMETIDO EM: 11/2019 ～～ACEITO EM: 11/2019 ｜ＰUBLICADO EM: 11/2019 


\section{RESUMEN}

Objetivo: Informe el caso clínico de un paciente diagnosticado con rancula y la técnica de tratamiento propuesta, donde mostró resolución para el caso. Detalle del caso: Paciente femenina, de 23 años, asistió al servicio, con la queja principal "Tengo una burbuja en mi boca que desaparece y regresa, y no duele". En la historia de la enfermedad actual, el paciente se queja de una lesión en forma de ampolla, que apareció hace aproximadamente seis meses, en la que a menudo aparece y desaparece, sin causa aparente, y esta lesión es indolora. En el examen intraoral, se observó una sola lesión ampollosa de color violeta, superficie lisa, límites claros, forma ovalada, flotante, indolora y ubicada en el piso vestibular. La hipótesis del diagnóstico fue de Ranula. Se realizó la marsupialización de la lesión y la lesión posterior se confirmó por histopatología. Consideraciones finales: Debido a que es un diagnóstico diferencial de otras lesiones, se realizó una histopatología para un diagnóstico correcto y la ejecución del tratamiento más apropiado para el caso, que sería la cirugía para extirpar el techo de la lesión, cuyo procedimiento tiene el mejor pronóstico. Es de gran importancia que el dentista obtenga conocimiento para una mejor conducta de tratamiento y especialmente la supervivencia del paciente.

Palabras clave: Mucocele, Ranula, Patología oral.

\section{INTRODUÇÃO}

As rânulas se apresentam como lesões nodulares, flutuantes e além disso, não possuem malignidade, sua cor pode variar do translúcido ao azulado, ou mesmo com a mesma coloração da mucosa oral, são pseudocistos retentivos, que se desenvolve no assoalho bucal, ocorrem geralmente através de um trauma nas glândulas sublinguais ou submandibulares ou devido a obstrução dos ductos salivares (NEVILLE BW, et al., 2016; EFFAT KG, 2012; AMARAL MB, et al., 2012).

Estas lesões são resultantes da liberação de mucina para o estroma, localizado no tecido conjuntivo, essa retenção surge com menos frequência, e é predominante em pessoas mais idosas, já o extravasamento de muco são mais comuns em crianças e adultos jovens (ARUNACHALAM P, et al., 2010). Existe uma proporção quanto a predileção pelo sexo feminino de $50,7 \%$ e sexo masculino de $49,3 \%$, quanto a região mais acometida, $60,6 \%$ das lesões são encontradas em lábio inferior (ZHI K, et., 2009; WANDERLEY FGC, et al., 2013).

Clinicamente as rânulas são fáceis de identificar, a dificuldade no diagnóstico se dá apenas, quando não existe um meio bucal adequado, dificultando a visualização pelo profissional, e quando isso ocorre, são lançados mão de exames complementares, como radiografias que evidencia material radiopaco, onde auxiliam na visualização do canal, ou exame de ultrassonografia que se é possível observar a extensão da lesão (AGUIRRE H, 2014).

Microscopicamente a lesão apresenta uma área de mucina extravasada, e presença de tecido de granulação reacional ao seu redor, com numerosos macrófagos de aspecto espumoso. Infiltrado inflamatório crônico e ductos dilatados normalmente são encontrados nas glândulas salivares menores adjacentes (NEVILLE BW, et al., 2016).

As rânulas costumam não apresentar sintomatologia dolorosa, porém a depender de seu tamanho, assim como de sua localização, quando chegam a ocupar a maior parte do assoalho bucal, causam uma elevação na região da língua e bastante desconforto ao paciente dificultando no momento de sua fala e deglutição (MARTINEZ L, et al., 2010).

Existem lesões que são auto limitantes, se rompem e cicatrizam sem a necessidade de intervenção (NEVILLE BW, et al., 2016), porém, esses casos não costumam ocorrer com frequência havendo a necessidade de tratamento (AMARAL MB, et al., 2012).

Várias são as técnicas cirúrgicas para o tratamento de rânulas, podemos citar, resseção por via cervical ou intra-oral, drenagem da lesão e também recessão juntamente com a glândula, excisão com laser de dióxido de carbono (CO2) e marsupialização (HABERAL I, et al., 2004), porém, fatores como idade do paciente, características, localização e profundidade da lesão interferem na hora da escolha desse tratamento (REGEZI JA, et al., 2017).

REAS/EJCH | Vol.Sup.37 | e2369 | DOI: https://doi.org/10.25248/reas.e2369.2019 Página 2 de 7 
A marsupialização é uma técnica menos traumática onde se utiliza de artifícios para manter o ducto da glândula salivar em comunicação com a cavidade oral, este método evita danos estruturais a tecidos vitais adjacentes (YAGUE GJ, et al., 2009). E a micromarsupialização é comumente utilizada em pacientes pediátricos, pois além de ser menos traumática possui uma chance menor da lesão recidivar (BAHARVAND M, et al., 2014).

Os prognósticos são satisfatórios, mesmo existindo uma menor possibilidade das lesões recidivarem após a utilização desta técnica (AMARAL MB, et al., 2012; NEVILLE BW, et al., 2016). Caso não seja feita uma abertura de janela cirúrgica de tamanho suficiente para impedir o fechamento da lesão, haverá novamente a união desse tecido e posterior acúmulo de líquido dentro do canal, na tentativa de evitar essa situação é de suma importância que haja uma ampla margem cirúrgica durante a execução da técnica (TEIXEIRA RG, et al., 2011; PINTO GNS, et al., 2015).

Diante do exposto, esse trabalho tem como objetivo relatar o caso clínico de uma paciente diagnosticada com rânula e a técnica de tratamento proposto onde o mesmo se demostrou de boa resolutividade para o caso.

\section{RELATO DE CASO}

Paciente, gênero feminino, 23 anos, feoderma, compareceu à Clínica Escola de Odontologia do Centro Universitário Cesmac, com queixa principal "Tenho uma bolha na boca, que desaparece e volta, ela não dói”. Na história da doença atual, a paciente se queixa de lesão em formato de bolha, que surgiu há aproximadamente seis meses, na qual surge e desaparece frequentemente, sem causa aparente e sem presença de sintomas dolorosos. Ao ser questionada a paciente relatou ter procurado tratamento pelo fato da lesão sempre tornar a aparecer.

Ao exame extra oral, a paciente não apresentava assimetria facial, quanto ao exame intra oral foi observado uma lesão bolhosa, única, medindo 2,5 × $1 \times 5 \times 1,0$ milímetros, de cor violácea, com superfície lisa, seus limites eram nítidos, de formato ovalado, lesão flutuante, sem presença de sintomatologia dolorosa estando localizada no assoalho bucal (Figura 1).

Figura 1 - Lesão unilateral.

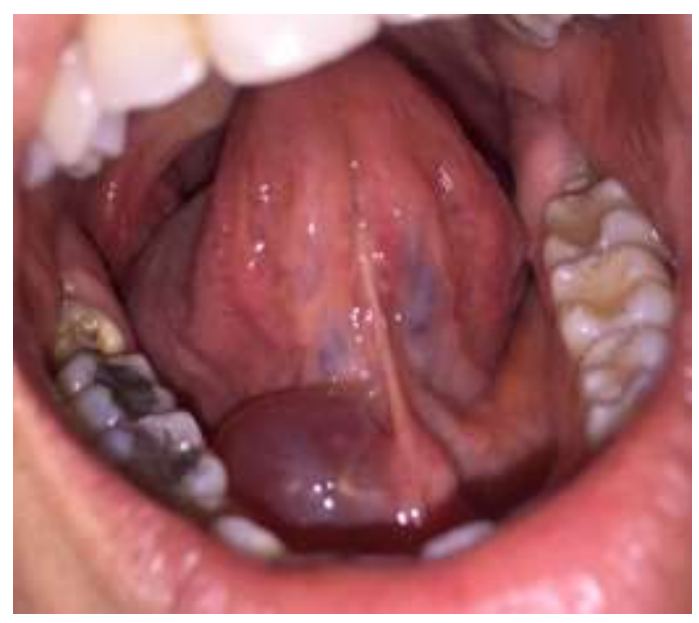

Fonte: GOMES FP, et al., 2017.

Após a confirmação do diagnóstico de rânula, como forma de tratamento foi escolhido a marsupialização, trata-se de uma técnica mais conservadora, e consequentemente menos invasiva, onde se confecciona uma janela cirúrgica que fica suturada próximo a mucosa bucal com o intuito de que haja uma comunicação entre a glândula e a cavidade oral. É removida a porção superior da lesão com preservação da glândula salivar sublingual. 
Para realização da marsupialização foi feito a antissepsia com clorexidina $0,12 \%$; anestesia local com lidocaína 2\% + epinefrina 1:100.000 na técnica infiltrativa no assoalho bucal, ao redor da lesão. A incisão da lesão foi realizada com lâmina de bisturi de número 15.C, evidenciando a excisão de uma porção da mucosa oral do assoalho bucal, juntamente com a parede superior da rânula, acarretando a saída de líquido salivar espesso e viscoso do interior da lesão, sendo representada na (Figura 2-A); apresentando uma loja cirúrgica desobstruída conforme mostra a (Figura 2-B).

Figura 2 (A e B) Marsupialização - remoção do teto da lesão e exposição da glândula.
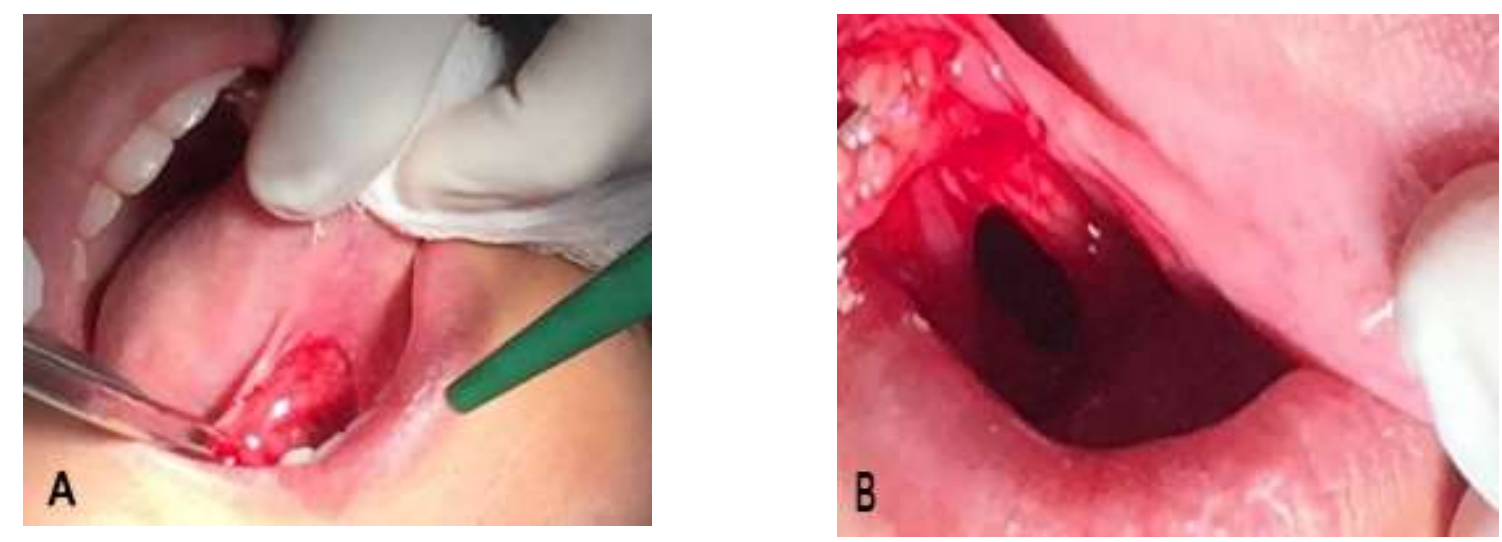

Fonte: GOMES FP, et al., 2017.

Posteriormente, foi recortado um pequeno pedaço de luva de procedimento para que esta exerça o papel de se manter como um dreno na região de dentro do canal onde havia muco, e a região foi suturada com fio de nylon número 0.3 milímetros para unir tecido de mucosa oral e a luva (Figura 3).Após a cirurgia foi recomendada a paciente todos os cuidados necessários, como alimentação pastosa, higienização da área com gaze e solução fisiológica com o intuito de remover toda alimentação impactada no momento da mastigação, afim de se evitar quaisquer tipos de injúrias evitando assim, uma maior contaminação na região onde foi realizado o procedimento cirúrgico. A paciente foi orientada na possibilidade de desconforto no pós cirúrgico a utilizar analgésicos.

Figura 3 - Artifício utilizado para simular ducto salivar.

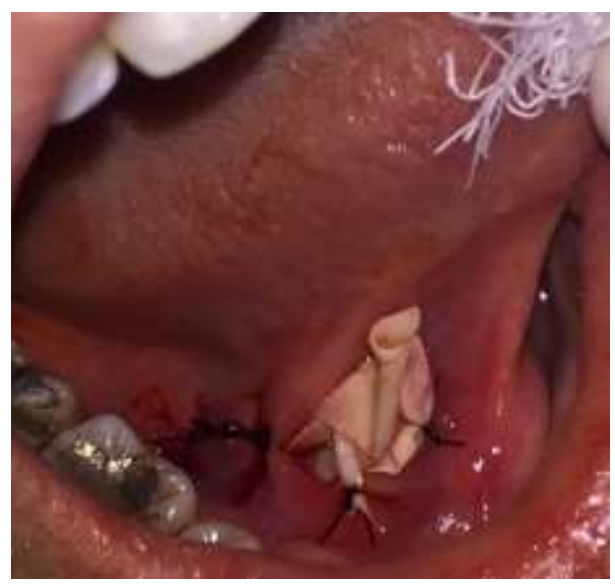

Fonte: GOMES FP, et al., 2017. 
Após a remoção da lesão, a peça foi encaminhada para o laboratório de patologia oral do Centro Universitário CESMAC para a realização do histopatológico. Na microscopia, no aumento de 40x foi observada a presença de tecido conjuntivo subjacente fibroso mostrado pela seta em preto, e presença de mucina extravasada indicada pela seta de cor verde na (Figura 4-A); já na microscopia de maior aumento de 100x foi observado intensa atividade proliferativa de macrófagos evidenciado pela seta azul, que são células de defesa responsáveis pelo controle de processo inflamatório, nesse caso atuando na contenção do extravasamento de mucina observado na (Figura 4-B).

Figura 4 A e B - Lâminas histológicas coradas em Hematoxilina e Eosina, apresentando semelhança a um cisto preenchida por muco e lâmina histológica mostrando mucina associada a proliferação de macrófagos, respectivamente.
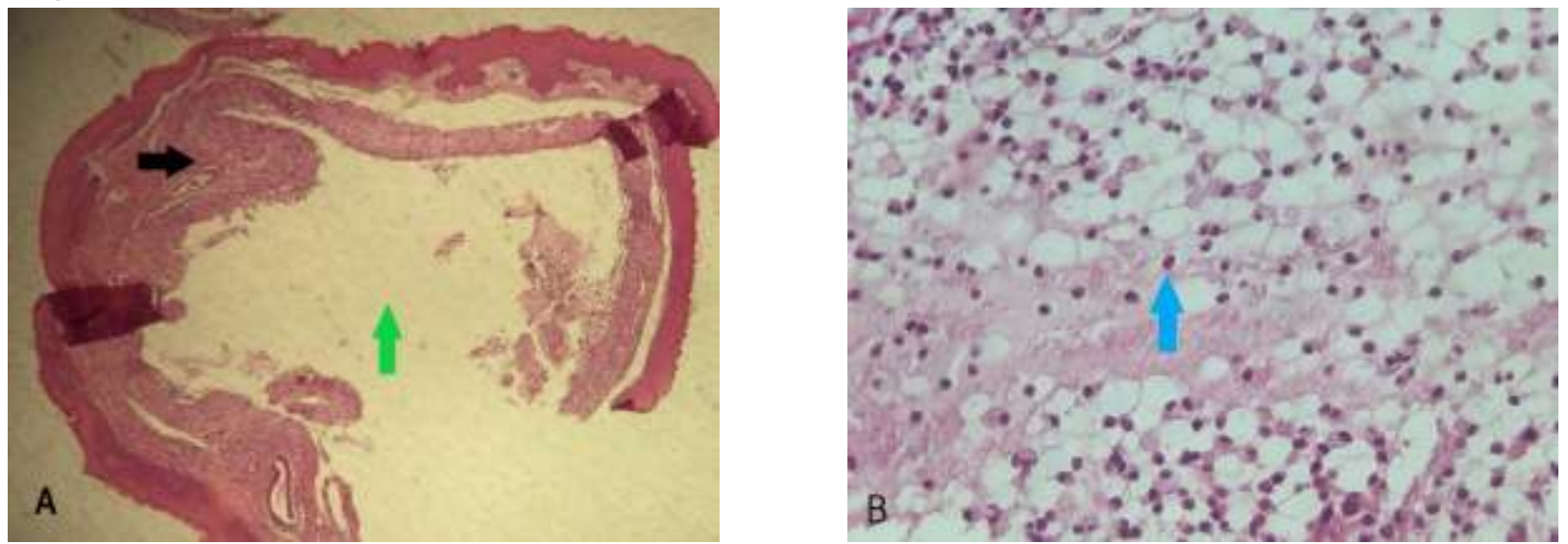

Fonte: GOMES FP, et al., 2017.

Após 28 dias, a paciente retornou para avaliação do pós cirúrgico, onde foi possível observar uma cicatrização satisfatória, sem recidiva da lesão, é importante que essa paciente seja acompanhada pela equipe que executou a cirurgia. Foi agendado um retorno após 6 meses, onde a mesma não apresentava nenhuma recidiva da lesão (Figura 6).

Figura 6 - Registro após 28 dias da cirurgia realizada na paciente (no qual podemos observar que não ocorreu recidiva).

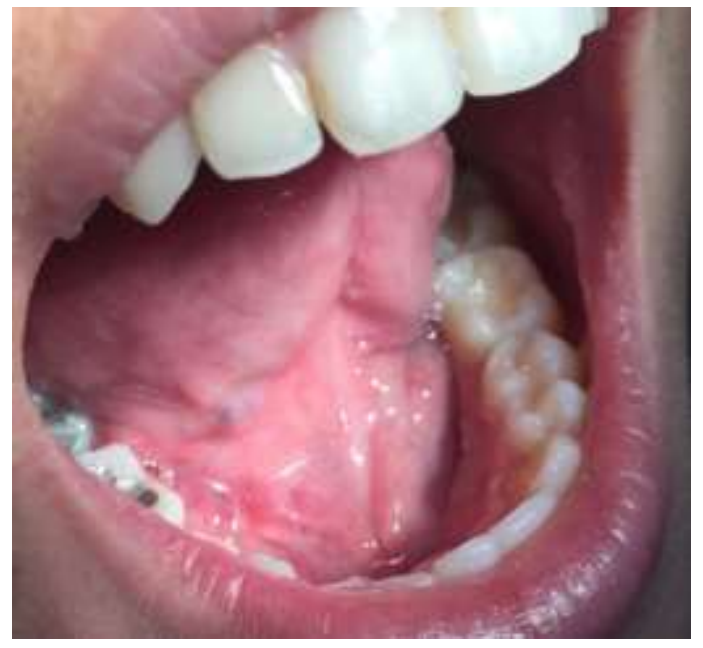

Fonte: GOMES FP, et al., 2017. 


\section{DISCUSSÃO}

Segundo a literatura, as rânulas são lesões que se localizam no assoalho bucal, possuem boa delimitação, normalmente é encontrada unilateralmente, sua coloração pode ser arroxeada e seu tamanho costuma variar e a depender de sua localização e dimensão, pode interferir na fala e deglutição, causando certo desconforto (ALQHTHANI N, et al., 2012). Sua evolução costuma ser rápida e quanto aos sintomas, são normalmente assintomáticos, se não apresentarem nenhum halo inflamatório e crescimento da lesão (LAWAL AO, et al., 2013).

Os artigos estudados mostram que a localização unilateral das lesões são vistas na maioria dos casos, porém algumas vezes podem ser confundidas como lesões bilaterais, pelo fato de atingir um volume tão grande que faz com que a lesão invada o espaço oposto, dando assim uma falsa impressão de bilateralidade na hora que é realizado o exame intra oral (LAWAL AO, et al., 2013).

No caso em questão a paciente apresentava uma lesão única sem causa aparente, formato bolhoso, tendo sua coloração violácea, relatando nenhum tipo de sintomatologia, nem quando estimulada, apenas o aparecimento constante da lesão, coincidindo assim com os relatos descritos nas literaturas estudadas.

A forma de tratamento das lesões de rânula, podem incluir técnicas cirúrgicas e não cirúrgicas (KIM JH, 2014; NIKITAKIS NG, et al., 2013; YAGUE GJ, et al., 2009). As cirúrgicas como a técnica de remoção da glândula sublingual, é vista por alguns autores como radical por mais que apresente menos recidiva, pode em algumas lesões a depender da localização, causar injúria como, por exemplo, sangramento no transoperatório e dificuldade na cicatrização (FRITZ GR, et al., 1997). O caso relatado não apresentou nenhuma intercorrência.

No caso apresentado, a marsupialização foi escolhida devido a técnica ser mais conservadora, simples, causar menos desconforto a paciente e a mesma, apresentar uma boa condição geral. Segundo a literatura, a escolha da técnica se dá, por ser menos invasiva e por não lesar os tecidos adjacentes (KIM JH, 2014).

Alguns autores enfatizam que embora a marsupialização seja uma técnica que apresente um bom prognóstico, deve-se observar por exemplo, a questão da higienização do local da cirurgia que é realizado pelo paciente no qual o profissional não tem controle sobre essa questão, e se o paciente não for colaborador, pode-se na região onde a técnica foi realizada se formar por meio de impacção alimentar uma área de contaminação e isso causar injúria pós operatória (TEIXEIRA RG, et al., 2011; PINTO GNS, et al., 2015).

Referente a esse caso a instituição segue o protocolo pós cirúrgico, onde o paciente recebe impresso todas as orientações pós operatórias incluindo cuidados na higienização do local (limpeza utilizando gaze e solução fisiológica 0,9\%), sempre após cada refeição e com os retornos da paciente a instituição foi possível observar que o local da cirurgia estava sendo bem higienizado e sem a presença de qualquer infecção.

Outros autores consideram que utilizando apenas esta técnica se consiga a resolução para o caso, levando em consideração o estado geral do paciente e sobretudo a faixa etária, diminuindo as chances de morbidade (TABRIZI R, et al., 2012). E em relação a idade da paciente do caso, uma adulta jovem, e segundo a literatura, ocorre em crianças e jovens, o que pode ser explicado devido ao fato de que nessas faixas etárias existem a maior probabilidade de traumatismos, causando assim, extravasamento de mucina (ZHI K, et., 2009; WANDERLEY FGC, et al., 2013).

Exames complementares são utilizados para que se feche o diagnóstico como, tomografias computadorizadas, ressonância magnética nuclear, ultrassonografia, citologia através de aspiração para posterior análise bioquímica (ALQHTHANI N, et al., 2012; EFFTAL KG, 2012). Entretanto, para este caso, o protocolo da instituição sobre esse tipo de lesão é a realização do histopatológico, e por esse motivo, não foram solicitados exames complementares.

Outras literaturas defendem a utilização da técnica de aspiração com agulha fina para que se obtenha um resultado mais preciso quanto ao diagnóstico (ALQHTHANI N, et al., 2012; EFFTAL KG, 2012), sendo essa técnica realizada através da observação da cor do líquido presente na lesão, que quando se encontra claro é considerada uma lesão menos inflamada e a lesão está recente, já nos casos onde a cor é observada mais escura 
(âmbar) suspeita-se de uma lesão crônica e com maior tempo de acometimento (ZHI K, et., 2009; WANDERLEY FGC, et al., 2013).

Para este caso o protocolo utilizado pela instituição foi de exame histopatológico que mostra a presença de mucina, assim como, das células presentes na lâmina histológica que definem a lesão e consequentemente resulta no diagnóstico.

Por ser diagnóstico diferencial de outras lesões, foi visto a melhor maneira de execução do tratamento para o caso, que seria a cirurgia para remoção do teto da lesão, sendo esse procedimento o que possui melhor prognóstico (NEVILLE BW, et al., 2016).

A paciente regressou ao serviço odontológico com uma semana após o procedimento para remoção de pontos, e com 28 dias para avaliação da cicatrização, e como protocolo, retornos semestrais com intuito de observar se houve recidivas, e nesse caso, não houve até o presente momento. É de grande importância que o Cirurgiãodentista obtenha conhecimento para uma melhor conduta do tratamento e sobretudo, sobrevida do paciente.

\section{REFERÊNCIAS}

1. AGUIRRE H. Uso de OK-432 (Picibanil) como alternativa no quirúrgica para el manejo de ránulas y mucoceles. The use of OK-432 (Picibanil) as a non-surgical alternative for the management of ranula and mucoceles. Revista ADM, 2011; 68 (5): 215-221.

2. ALQHTHANI N, et al. Ranula: pathogenesis and management - A review. Int J Dent, 2012; 11(1): 49-54.

3. AMARAL MB, et al. Upgrading of the micromarsupialisation technique for the management of mucus extravasation or retention phenomena. Int J Oral Maxillofac Surg, 2012; 41(12): 1527-1531.

4. ARUNACHALAM P, et al. Recurrent plunging ranula. J Indian Assoc Ped Surg, 2010; 15(1): 36-38.

5. BAHARVAND M, et al. Treatment of labial mucocele by intralesional injection of dexamethasone: case series. J Dent Mater Tech, 2014; 3(3): 128-133.

6. EFFAT KG. Acute presentation of a plunging ranula causing respiratory distress: case report. J Laryngol Otol, 2012; 12(6): 861-863.

7. FRITZ GR, et al. Complications following mucous cyst excision. J Hand Surg, 1997; 22(2): 222-225

8. HABERAL I, et al. Surgical management of pediatric ranula. Int J Pediatr Otorhinolaryngol, 2004; 68(2): 161-163.

9. JIA Y, et al. Clinical and histopathological review of 229 cases of ranula. J Huazhong Univ Sci Technol, 2011; 31(5): 717-720.

10. $\mathrm{KIM} \mathrm{JH}$. Ultrasound-guided sclerotherapy for benign non-thyroid cystic mass in the neck. Ultrasonography. 2014; 33(2): 83- 90.

11. LAWAL AO, et al. A review of 413 salivary gland tumours in the head and neck region. J Clin Exp Dent, 2013: 5(5): 218222.

12. MARTINEZ L, et al. Clinical Characteristics, Treatment, and Evolution of 89 Mucoceles in Children. Oral Surgery and Implantology, 2010; 68(10): 2468-2471.

13. NEVILLE BW, et al. Patologia Oral e Maxilofacial, 2016; 4. ed. Rio de Janeiro: Elsevier.

14. NIKITAKIS NG, et al. Asymptomatic swelling in the floor of the mouth. Salivary duct cyst (ranula). Gen Dent, 2013; 61(1): 77-78.

15. PINTO GNS, et al. Marsupialização como tratamento definitivo de cistos odontogênicos: relato de dois casos. RFO, 2015; 20(3): 361-366.

16. REGEZI JA, et al. Patologia bucal: correlações clinicopatológicas,2017; 7. ed. Rio de Janeiro: Guanabara Koogan.

17. TABRIZI R, et al. Marsupialization as treatment option for the odontogênico keratocyst. J Craniofac Surg 2012; 23(5): 459-461.

18. TEIXEIRA RG, et al. Decompression of a maxillary dentigerous cyst. Rev Gaúcha Odontol 2011; 59(2): 299-303.

19. WANDERLEY FGC, et al. Retrospective study on the prevalence of mucus retention and extravasation phenomenaclinical and histopatological correlation. RFO, 2013; 18(3):307-311.

20. YAGUE GJ, et al. Treatment of oral mucocele-scalpel versus CO2 laser. Med Oral Patol Oral Cir Bucal, 2009; 1(4): 469474.

21. ZHI K, et al. Management of the pediatric plunging ranula: results of 15 years clinical experience. Oral Surg Oral Med Oral Pathol Oral Radiol Endod, 2009; 107(4): 499-502. 